\title{
Motor Unit Behavior in Canine Motor Neuron Disease
}

\author{
M. J. Pinter, ${ }^{1}$ R. F. Waldeck, ${ }^{1}$ N. Wallace, ${ }^{1}$ and L. C. Cork ${ }^{2, a}$ \\ 'Department of Anatomy and Neurobiology, Medical College of Pennsylvania, Philadelphia, Pennsylvania 19129 \\ and ${ }^{2}$ Division of Comparative Medicine, Johns Hopkins University School of Medicine, Baltimore, Maryland 21205
}

\begin{abstract}
Hereditary canine spinal muscular atrophy (HCSMA) is an autosomally dominant disease of motor neurons that shares many pathological features with human motor neuron disease. A particularly striking feature of the affected, accelerated phenotype (homozygous HCSMA) is that profound weakness develops before appreciable motor neuron cell death occurs (Cork et al., 1989a), implying that motor unit functional defects occur initially. The purpose of this study was to identify the site of these defects and characterize their nature. In most young homozygotes (2-3 months postnatal), motor neurons were encountered that could support orthodromic action potential propagation to the muscle but did not activate muscle fibers. The tetanic forces of innervated motor units in young homozygotes tended to be smaller than those in closely age-matched clinically normal animals. In older homozygotes $(\approx 4.5$ months, postnatal), all motor neurons sampled were capable of activating muscle fibers, but many motor units displayed abnormal behavior including an inability to sustain force output during high frequency activation. Motor units exhibiting tetanic failure also showed proportionately greater twitch potentiation than nonfailing units of similar unpotentiated twitch amplitude. Tetanic failure and large potentiation tended to occur in motor units that possessed the slowest contraction speeds. These results indicate that motor neuron functional defects in HCSMA appear initially in the most distal parts of the motor axon and involve defective neurotransmission. The possible roles of distal nerve degeneration, motor terminal sprouting, and synaptic transmission in causing these deficits are considered.

[Key words: motor unit, motoneuron disease, neuromuscular junction, muscle, electrophysiology]
\end{abstract}

Hereditary canine spinal muscular atrophy (HCSMA) is an autosomal dominant disorder that selectively affects motor neurons (Cork et al., 1979, 1981; Sack et al., 1984). Individuals that are homozygous for the trait have a stereotyped clinical course. Weakness first appears at $6-8$ weeks of age and eventually pro-

Recejved Sept. 21. 1994; revised Nov. 7, 1994: accepted Nov. 10, 1994

We thank Dr. Donald Faber for helpful comments on the manuscript and Mark Dubrow for assistance during several experiments. We gratefully acknowledge the support of the National Institutes of Health (Grants NS3162I. NS24707. NS(7287). The HCSMA breeding colony was supported by PHS Grant NS10580 to Dr. Donald L. Price.

Correspondence should be addressed to Dr. Martin J. Pinter, Deparment of Anatomy and Neurobiology. Medical College of Pennsylvania. 3200) Henry Avenue, Philadelphia, PA 19129.

- Present address: Department of Comparative Medicine, Stanford University School of Medicine. Stanford. CA 94305-5410.

Copyright $\bigcirc 1995$ Society for Neuroscience $0270-6474 / 95 / 153447-11 \$ 05.00 / 0$ gresses to tetraparesis. Death may ensue as early as $21-22$ weeks unless supportive methods are used. The distribution of muscular involvement is bilaterally symmetrical and progresses from caudal to rostral and from proximal to distal. Thus, the first indications of involvement are observed in tail muscles. As in human motor neuron disease, oculomotor muscles and vesicorectal sphincters are spared. Evidence of sensory or cerebellar involvement has not been detected.

The neuropathologic features of HCSMA include proximal axonal swellings late in the clinical course of homozygous individuals. The motor roots in HCSMA homozygotes and heterozygotes fail to reach normal caliber and motor axons of adult HCSMA heterozygotes undergo atrophy, but there is no evidence of Wallerian degeneration in spinal roots (Cork et al., 1989b). The transport of neurofilaments, a major determinant of axonal caliber (Hoffman et al., 1984), and of tubulin is decreased, but fast transport appears unimpaired (Griffin et al., 1982). Quantitative in situ hybridization reveals a selective decrease in mRNA for one neurofilament subunit (NF-L) but not for other subunits (NF-M, NF-H) or polyA ${ }^{+}$in homozygotes (Muma and Cork, 1993). The relationship of these changes to the primary genetic defect and its principal pathological mechanism in HCSMA is unknown.

An intriguing feature of homozygote individuals is that severe weakness develops well before any signs of significant motor neuron cell death appear (Cork et al., 1989a). This suggests that motor unit functional deficits must precede motor neuron cell death in HCSMA. We reasoned that identifying the locations of these functional deficits, as well as clarifying the timing of their appearance relative to the progress of the disorder, could provide additional clues about pathological mechanisms operating in HCSMA. To accomplish this, we have used intracellular recording from HCSMA spinal motor neurons to functionally isolate single motor units for study. We present in this report initial findings from young homorygous individuals demonstrating that motor unit functional deficits appear first in the most distal parts of motor axons that are otherwise electrophysiologically competent. We also show that motor unit deficits of a different type appear in somewhat older homozygotes and that the likely site of these deficits remains localized to the distal motor axon.

A preliminary report of some of these results has appeared (Pinter et al., 1992).

\section{Materials and Methods}

\section{Genotype and phenotype}

All animals used in this study were obtained from an HCSMA breeding colony. The homozygous HCSMA (accelerated) phenotype has remained consistent for $>15$ years and can be readily identified by the appearance of weakness in the tail muscles beginning about 6-8 weeks of age. Prior to the onset of clinical signs (usually by 1 year of age), 
HCSMA heterozygotes, which later develop intermediate or chronic disease, are usually clinically indistinguishable from their normal siblings (Sack et al., 1984). Thus, the littermates of the HCSMA homozygotes used in this study were all phenotypically clinically normal, but genetically were either homozygous normal or heterozygous HCSMA.

\section{Surgical preparation}

Animals were initially anesthetized with $35 \mathrm{mg} / \mathrm{kg}$ intravenous sodium pentobarbital. Supplemental doses were administered during the experiment via an intravenous cannula to maintain an absence of withdrawal and corneal reflexes. A tracheal cannula was inserted to maintain a patent airway and provide for monitoring of end-tidal $\mathrm{CO}_{2}$. Blood pressure was continuously monitored via an arterial cannula. Rectal temperature was monitored and maintained at $37-38^{\circ} \mathrm{C}$ with a heating pad and infrared lamps.

The left medial gastrocnemius (MG) muscle and its nerve were exposed, and the muscle and its tendon were dissected free of the accompanying lateral gastrocnemius muscle and surrounding connective tissue as much as safely possible and prepared for attachment to a strain gauge for motor unit force recording. The strain gauge was capable of detecting forces of about $100-200 \mathrm{mg}$. All force recording was performed using a servo-operated device that maintained muscle preload at 100 $\mathrm{gm}$. The MG nerve was freed of surrounding tissue and mounted on a pair of bipolar stimulating/recording electrodes. Fine stainless steel wires (2-4 pairs) were inserted into the MG muscle and connected to high gain differential amplifiers for recording motor unit electromyographic (EMG) activity. A laminectomy was performed to expose the lumbosacral spinal cord. The animal was then mounted in a frame that immobilized the vertebral column and the left hindlimb. Exposed tissues were covered with warm mineral oil or Vaseline.

At the conclusion of surgical preparations, all animals received bilateral pneumothorax and were mechanically respirated to minimize respiratory-associated movements during intracellular recording. All animals remained unparalyzed.

\section{Recording procedures}

Single MG motor neurons were identified by antidromic activation following electrical stimulation of the $\mathrm{MG}$ nerve. Intracellular records were obtained using nicropipelles filled with $3 \mathrm{M} \mathrm{KCl}$ and connected to a conventional amplifier. Following impalement of an MG motor neuron, data were collected for measuring and analyzing motor axon conduction velocity as well as several other motor neuron electrical properties as described previously for cat (Pinter et al., 1991). Detailed analyses of motor neuron electrical properties and their relationships with motor unit mechanical properties will be the subject of another report.

Motor unit properties. Single motor units were activated using 0.5 msec depolarizing current pulses delivered through the micropipette. Motor unit twitch data (twitch contraction force and time to peak) were collected first. The twitch contraction was examined for posttetanic po tentiation (PTP) by alternating single twitch contractions with short trains of stimuli delivered at $150 \mathrm{~Hz}$. Fused tetanic contractions were then collected for stimulus trains of 100 and $200 \mathrm{H} z$. Sone HCSMA homozygote motor units failed to sustain fused contractions during tetanic stimulation. Tetanic force in these units was measured as both the peak force and force present at the end of the stimulus train. Motor unit fatigue was tested with short trains of depolarizing pulses $(13$ pulses at $40 \mathrm{~Hz}$ ) delivered at a frequency of $1 \mathrm{~Hz}$ for a $2 \mathrm{~min}$ period (Burke et al., 1973).

Some motor units failed to exhibit force following intracellular stimulation of MG motor neurons. In these cases, the motor neuron was first tetanized to insure the absence of measurable motor unit force. To detect the presence of motor unit EMG activity, the motor neuron was then stimulated at $15 \mathrm{H} /$ and up to 300 sweeps of EMG signals from each of the two to four intramuscular electrode pairs were obtained and averaged. In some cases, simultaneous averaging of single motor axon action potentials was obtained from the peripheral stinulating electrode

\section{Results}

A total of seven homozygotes from four inbred litters was studied (Table 1). Five of these animals were studied 66-87 d postnatal (younger homozygotes), while the remaining two were studied about $130 \mathrm{~d}(128,136)$ postnatal (older homozygotes).

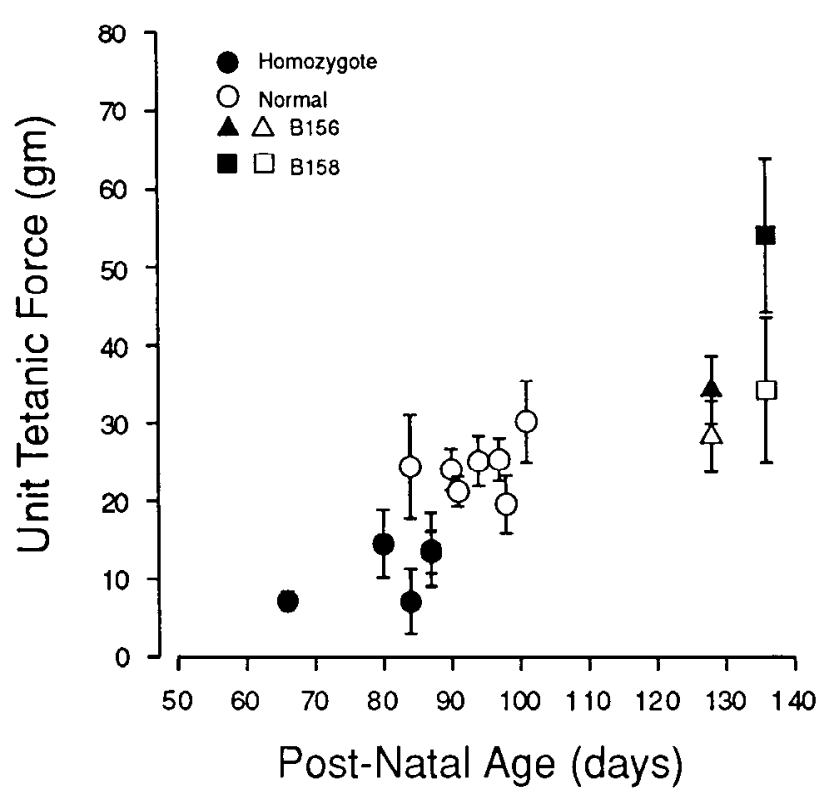

Figure 1. Plot of mean motor unit telanic force versus postmatal age. Filled and open circles represent homozygotes and clinically normal animals, respectively. Note the presence of two mean values at $87 \mathrm{~d}$ postnatal. Because some units from older homozygotes (rightmost data) failed to maintain tetanic forces, two mean values are shown for each animal. These values represent the average peak tetanic force (filled triangle, square) and the average tetanic force present at the conclusion of the stimulus train (open triangle, square). Error bars indicate \pm 1 SEM.

The clinical status of all homozygotes included an inability to raise the head to an erect posture or stand for more than $30-60$ sec due to paralysis of neck and pclvic girdle muscles, atrophy of lumbar paraspinal muscles, and marked paralysis of the tail. None of these animals had become tetraparetic. The appearance of these symptoms at this early age defines the homozygote phenotype. We also studied seven clinically normal animals aged 84-101 d postnatal. Note in Table 1 that six of these animals were littermates of investigated homozygotes and were thus either HCSMA heterozygotes or homozygous normal.

\section{Younger homozygotes}

We encountered two types of motor neurons in younger homozygotes. Both types could be antidromically activated following electrical stimulation of the $\mathrm{MG}$ nerve about $1 \mathrm{~cm}$ proximal to its entry into the muscle. In the first type, observed in all five young homozygotes, intracellular stimulation produced measurable motor unit forces. As shown in Figure 1, mean motor unit tetanic forces from these animals (filled circles, aged 66-87 d) were smaller than those obtained from clinically normal littermates (open circles, aged 84-101 d). Restricting consideration to these two groups and using mean values only, both age and phenotype contribute to this difference since these factors were significantly correlated with unit tetanic force (vs age, $r=0.76$; vs phenotype, $r=0.89 ; p<0.01$ in both cases) as well as with each other $(r=0.69, p<0.01)$. To address the relative contributions of these factors, we performed a partial correlation analysis on mean unit tetanic force in which the correlations of age or phenotype with force could be examined while holding the other factor constant (Hays, 1988). This analysis showed that phenotype was better correlated with tetanic force $(r=0.78, p$ $<0.001$; age held constant $)$ than age $(r=0.43, p>0.05$; 
Table 1. Summary of motor neuron connectivity

\begin{tabular}{llllllr} 
Study & Type & Litter & Age“ (days) & $\begin{array}{l}\text { Unconnected } \\
\text { units }\end{array}$ & $\begin{array}{l}\text { Connected } \\
\text { units }\end{array}$ & $\begin{array}{l}\text { Total } \\
\text { units }\end{array}$ \\
\hline B134 & Homozygote & 1 & 66 & 19 & 7 & 26 \\
B139 & Homozygote & 1 & 87 & 4 & 8 & 12 \\
B145 & Homozygote & 2 & 84 & 3 & 6 & 9 \\
B148 & Homozygote & 2 & 87 & 4 & 13 & 17 \\
B156 & Homozygote & 3 & 136 & 0 & 29 & 29 \\
B158 & Homozygote & 3 & 128 & 0 & 20 & 20 \\
B170 & Homozygote & 4 & 80 & 0 & 11 & 11 \\
M20. & Normal & na & 84 & 0 & 8 & 8 \\
B135 & Normal & 1 & 101 & 0 & 19 & 19 \\
B136 & Normal & 1 & 97 & 0 & 20 & 20 \\
B137 & Normal & 1 & 90 & 0 & 23 & 23 \\
B140 & Normal & 1 & 94 & 0 & 15 & 15 \\
B147 & Normal & 2 & 98 & 0 & 14 & 14 \\
B151 & Normal & 2 & 91 & 0 & 11 & 11 \\
\hline
\end{tabular}

"A1 these ages, heterozygous HCSMA and normal animals are clinically indistinguishable.

"Units are included only if motor unit fore could be tested by single pulse stimulation of motor neurons and unit EMG averaging or single pulse/tetanic stimulation and unit force recording.

Animal M20 was from an unrelated pedigree.

phenotype held constant). These results demonstrate that $\mathrm{MG}$ unit forces are smaller in these young homozygotes than in the young clinically normal animals independent of age. Other unit mechanical properties from younger homozygotes were similar to clinically normal animals.

The second type of antidromically identified motor neuron appeared not to be connected functionally to muscle fibers. Such motor neurons were encountered in four of five young homozygotes but not in clinically normal animals or in older homozygotes (Table 1). Single pulse stimulation of these motor neurons did not produce twitch responses, and high frequency stimulation also failed to provoke force output. Records from implanted EMG wires were also averaged while delivering in-

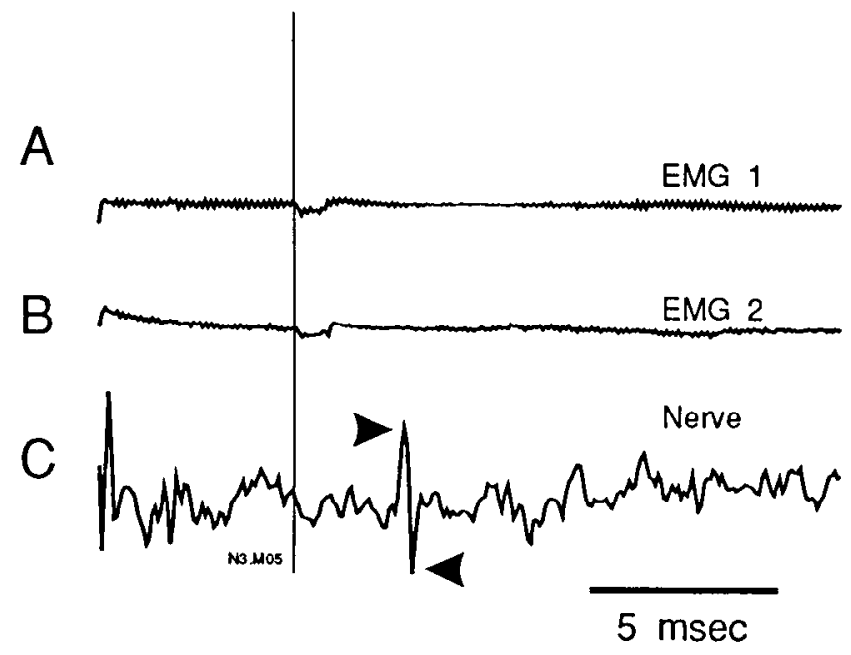

Figure 2. Example of single motor axon action potential in the absence of EMG activity in a young homozygote. All signals obtained simultaneously during intracellular stimulation of a single motor neuron. $A, B$, Averaged records (300)) of motor unit EMG activity taken from two locations in the $\mathrm{MG}$ muscle. $C$. Averaged record of $\mathrm{MG}$ nerve activity. The recording site was about $1 \mathrm{~cm}$ proximal to the entry of the nerve into the muscle. The onset of intracellular stimulation of the MG motor neuron is indicated by the vertical line. tracellular stimuli, but these did not reveal evidence of muscle fiber activation that might have been too weak to detect with the strain gauge. We considered the possibility that some HCSMA axons might not be capable of supporting orthodromic propagation to the periphery despite being capable of supporting antidromic conduction from peripheral stimulation sites. Thus, in addition to EMG records, records were obtained from peripheral nerve stimulation sites while stimulating motor neurons intracellularly in the second pair of younger homozygotes we studied (litter 2, Table 1). Figure 2 shows an example of averaged records obtained from one of these animals. In this case, intracellular stimulation of the motor neuron caused a biphasic axonal action potential at a latency consistent with the conduction velocity of this neuron, but no associated muscle electrical activity appeared. The biphasic nature of the averaged axonal signal indicates that the action potential swept past the differential recording leads. Results such as these were obtained in six of seven antidromically identified MG motor neurons that did not produce motor unit force upon intracellular stimulation and in which conditions allowed a sufficient number $(300)$ of records to be averaged. Table I shows that these "disconnected" motor neurons constituted $24-73 \%$ of the sampled unit population among younger homozygotes. It should be noted that the animal (B134) in which $73 \%$ of the population was "disconnected" was also the most severely affected young homozygote. These results demonstrate that some young homozygote motor neurons are incapable of activating muscle fibers despite being able to propagate action potentials to the periphery.

A failing motor unit. "Disconnected" motor neurons may be cells that initially extend axons to the level of the muscle but for some reason form few or possibly no functional synaptic contacts with muscle fibers. Alternatively, these may be neurons that form neuromuscular contacts with muscle fibers but eventually lose at least the functional capabilities associated with this contact. A case of a motor neuron from animal B134 that was initially capable of activating muscle fibers but lost this during high frequency stimulation supports the latter possibility.

As shown in Table 1 , only seven of 26 identified motor neu- 
A
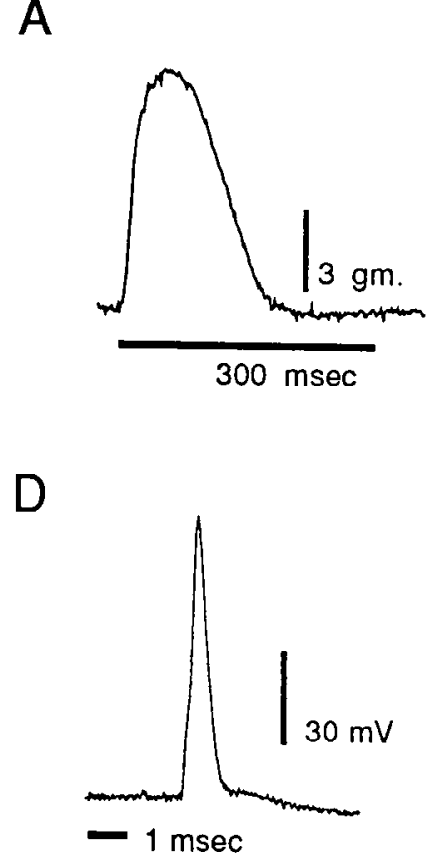

B

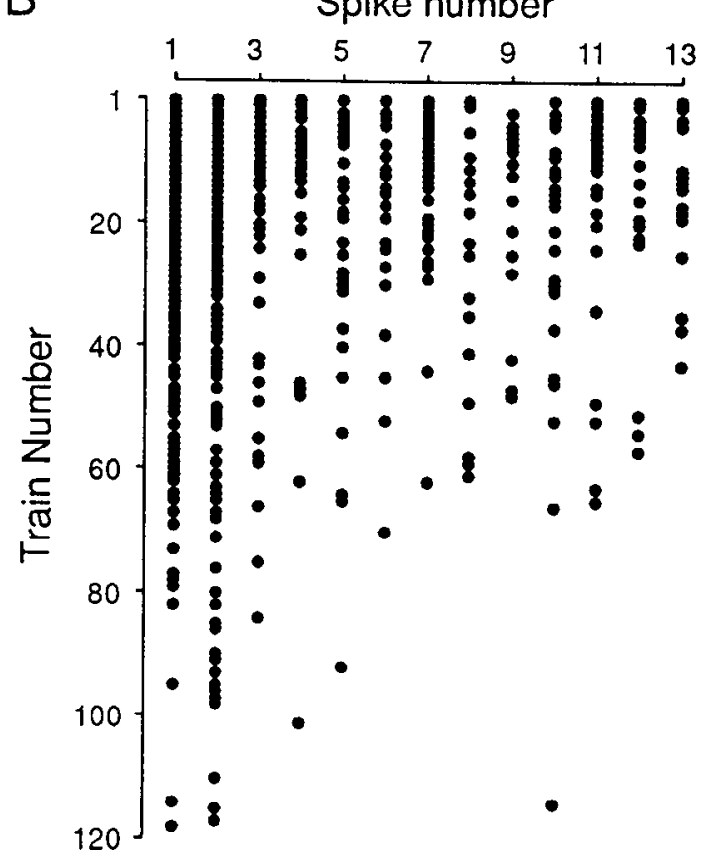

C
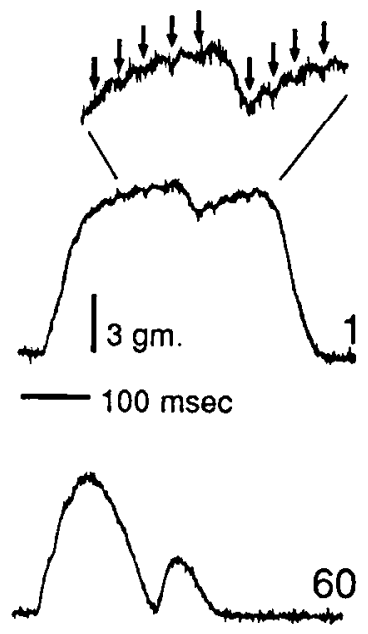

120

Figure 3. Failure of a single motor unit. The illustrated records were obtained from a motor unit that failed during tests for tetanic force and failed further during standardized fatigue test. A, Tetanic force. The motor neuron was stimulated intracellularly at $200 \mathrm{H} z$ for the duration indicated by the bar bolow the record. Note that force develops initially, but is not sustained. $B$. Each dot in the rasterplot indicates the occurrence of a motor unit contraction during trains of 13 depolarizing pulses $(40 \mathrm{~Hz})$ delivered to the motor neuron. (The topmost record in $C$ illustrates how individual twitch contractions were identified.) The train number $(120$ total at $1 \mathrm{train} / \mathrm{min})$ is plotted on the ordinate while the spike number for each train is plotted on the abscissa. Note increasing failure of motor unit response as fatigue test progressed. $C$, Sample records taken from the indicated train numbers. $D$. Antidromic action potential obtained after completion of motor unit testing, demonstrating the presence of antidromic conduction.

rons in B 134 produced detectable motor unit forec when stimulated intracellularly. While recording from one of these seven units, the motor unit began failing to follow the evoked activity and the failure seemed exacerbated by our testing. Force failure was first detected during the test for motor unit tetanic force in which the motor neuron was stimulated with $200 \mathrm{H} z$ trains of $0.5 \mathrm{msec}$ depolarizing pulses (Fig. $3 A$; intracellular pulse train duration is indicated by the 300 misec bar below the force trace) While the unit developed a fused tension profile initially, this was not maintained for the duration of the stimulus train. The possibility that the motor neuron itself failed to fire during the train was excluded by oscilloscope monitoring.

The unit was next examined for fatigue, in which the motor neuron was stimulated for 2 min with trains of 13 pulses (at 40 $\mathrm{H} \%, 1 \mathrm{train} / \mathrm{sec}$ ). The rasterplot in Figure $3 B$ illustrates the behavior of the unit during this test. Each dot in Figure $3 B$ signifies the occurrence of a unit twitch contraction in response to a single motor neuron depolarizing pulse. The abscissa represents each of 13 pulses per train with the train number plotted on the ordinate. Initially, little failure was evident with almost all (12 of 13) motor neuron stimuli producing unit contractions (Fig. 3C, trace 1). The failure gradually increased and was complete at the conclusion of the test (Fig. 3C, trace 120). Figure $3 D$ illustrates an antidromic action potential obtained at the conclusion of the fatigue test, demonstrating that retrograde axonal action potential propagation remained intact.

Averages of intracellular, unit force, and unit EMG signals show that the force failure observed during the fatigue test was associated with a corresponding failure of unit EMG but not with failure of motor neuron activation. For each of the 13 motor neuron spikes, the raster data of Figure $3 B$ were used to identify trains in which unit force appeared or failed. The corresponding intracellular, unit force, and EMG signals were then combined into two groups and averaged separately. The resulting set of averages for the first spike in the train is illustrated in Figure 4. Column A shows intracellular (top row), unit EMG (middle), and unit force (bottom row) averages for sweeps in which unit force appeared in response to the first spike (indicated by arrow in top row), while column B shows corresponding records for sweeps in which unit force did not appear. The intracellular records are clipped to show only the average afterhyperpolarization (AHP). It may be seen that the absence of unit force related to the first spike in Figure $4 B$ is associated with a complete absence of unit EMG. The averaged AHPs in each column are nearly identical, however, as would be expected if the motor neuron spike did not fail. Similar results were obtained for the remaining spikes in the train.

These results indicate that force failed in this unit because of conduction failure in the motor axon and that the likely site of failure is the distal axon. This is supported by the fact that unit force and EMG failure were associated and that antidromic activation from the distal nerve stimulation site remained intact at the conclusion of the test. The data also suggest that this failure was increased by the imposed activity of our testing. It may be that such activity-related axonal conduction block is the crucial factor that leads to the phenomenon of "disconnected" motor neurons.

\section{Older HCSMA homozygotes}

Results obtained from the pair of older homozygotes differed from those obtained from younger HCSMA homozygotes. In 


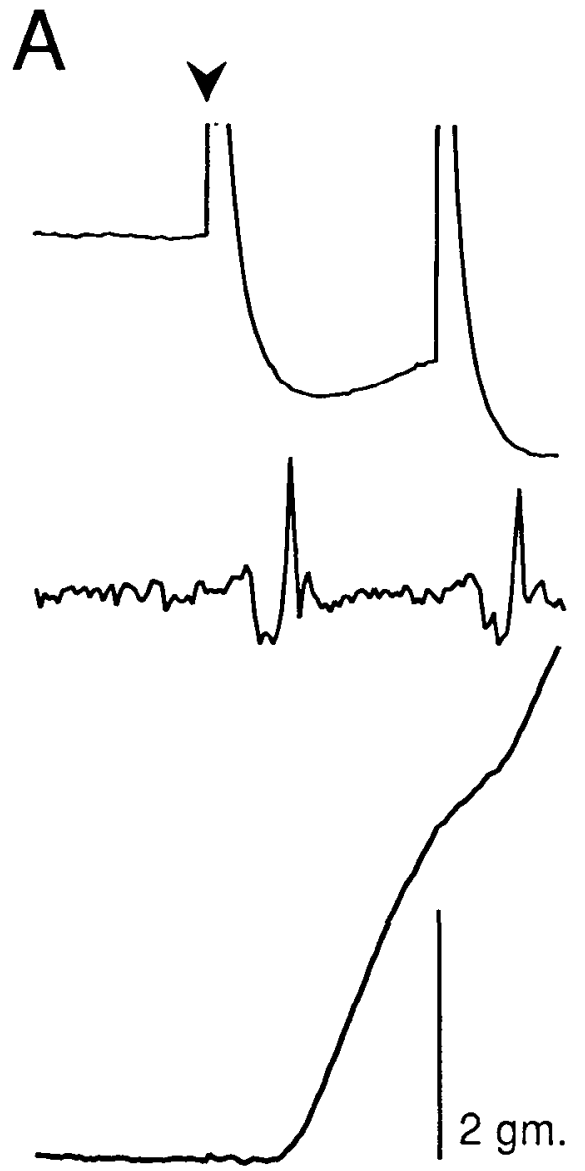

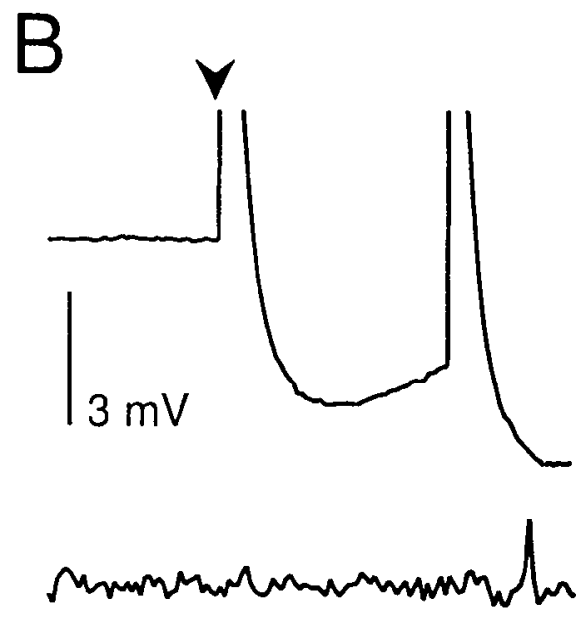

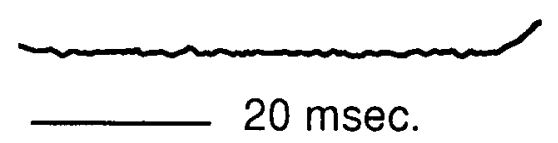

Figure 4. Motor unit failure during fatigue test involves both EMG and force failure but not spike failure. $A$ shows averages of intracellular (top), EMG (middle), and force (bottom) records for all fatigue test trains in which the first spike of the train (indicated by arrowhead in top record) produced a force response $(N=79)$. $B$ shows corresponding averaged records for all trains in which no twitch force appeared following the first spike $(N=$ 41). The intracellular averages are clipped to show motor neuron AHP only. The records demonstrate that unit force failure occurred independent of associated motor neuron spike occurrence and that both unit force and EMG are absent when unit force failure occurs. this group, all antidromically identified $\mathrm{MG}$ motor neurons were capable of activating muscle fibers following intracellular stimulation. However, the functional properties of many motor units were abnormal, the principal defect being an inability to maintain tetanic force output. An example of this is illustrated in Figure 5 . In Figure $5 A$, the motor neuron was stimulated with a train $(100 \mathrm{~Hz})$ of depolarizing pulses, with the train duration indicated by the heavy bar. As can be seen, the initial level of evoked EMG activity is promptly followed by a decline of unit EMG amplitude and a corresponding decline of unit force. The association of force with EMG failure indicates that the number of active muscle fibers decreases as the train proceeds and sug gests that a failure of neurotransmission is likely to be involved. The fact that a reduced level of unit EMG activity is sustained to the conclusion of the stimulus train demonstrates that transmission to some muscle fibers remains suprathreshold but that this capability is nonuniformly distributed among all initially active connections made by this motor neuron. Figure $5 B$ shows, however, that failure of unit force and EMG was nnarkedly increased at $200 \mathrm{~Hz}$ stimulus frequency, with EMG activity being indistinguishable from baseline noise at the conclusion of the stimulus train.

Tetanic failure was quantified by taking the difference between the initial peak tetanic force and force at the end of the tetanus (arrowheads, Fig. $5 A, B$ ) and normalizing the value to the peak force. The bar graphs in Figure $5 C$ show that increasing motor neuron stimulus frequency from $100 \mathrm{~Hz}$ to $200 \mathrm{~Hz}$ in- creased failure on average by about $15 \%$ in one and about $17 \%$ in the other experiment.

The occurrence of tetanic force failure was quite common, being present in 9/21 units in one experiment and 10/14 in the other. Tetanic failure was also very selective for the older homozygotes; the example illustrated in Figure 3 is one of only two cases observed in all five younger homozygotes, and such failure was not observed in clinically normal animals.

The overall mean unit tetanic forces $(200 \mathrm{~Hz}$ ) for the older homozygotes are shown plotted against postnatal age in Figure 1 (four rightmost values). The upper data points (filled symbols) represent mean peak force of failing units, while the lower means (open symbols) include forces present at the end of the stimulus trains. It may be seen that when tetanic failure is taken into account, the population means are displaced into the range of younger, clinically normal animals.

Motor unit twitch potentiation. A number of units that displayed tetanic failure also exhibited relatively large potentiation of twitch amplitude following the first few delivered tetani. Several units that exhibited the largest tetanic failure and twitch potentiation provided evidence that the conditioning tetani activated additional muscle fibers and that a single high frequency train could be sufficient to provoke the effect. An example of this is shown in Figure 6 . The initial twitch contraction force of this unit, observed before any repetitive stimuli were used, was quite small but nonetheless detectable (Fig. 6A). The first high frequency $(150 \mathrm{~Hz})$ train of stimuli delivered to the motor neu- 
A $100 \mathrm{~Hz}$.

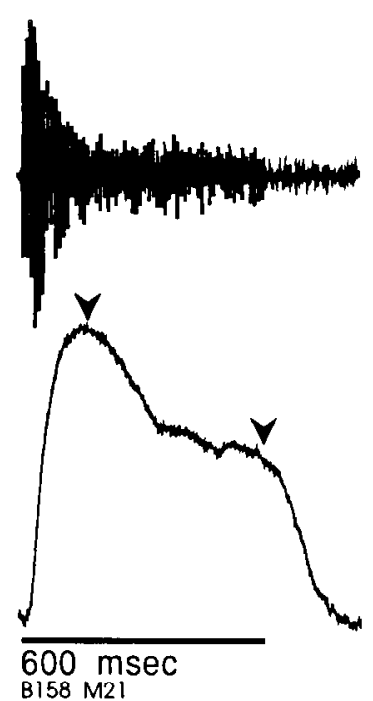

B $200 \mathrm{~Hz}$.

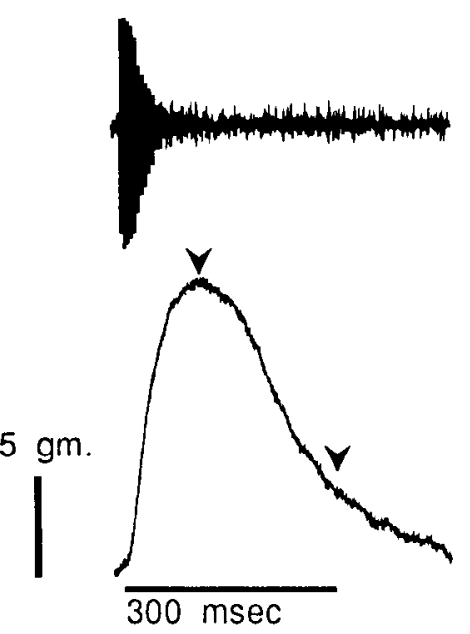

C

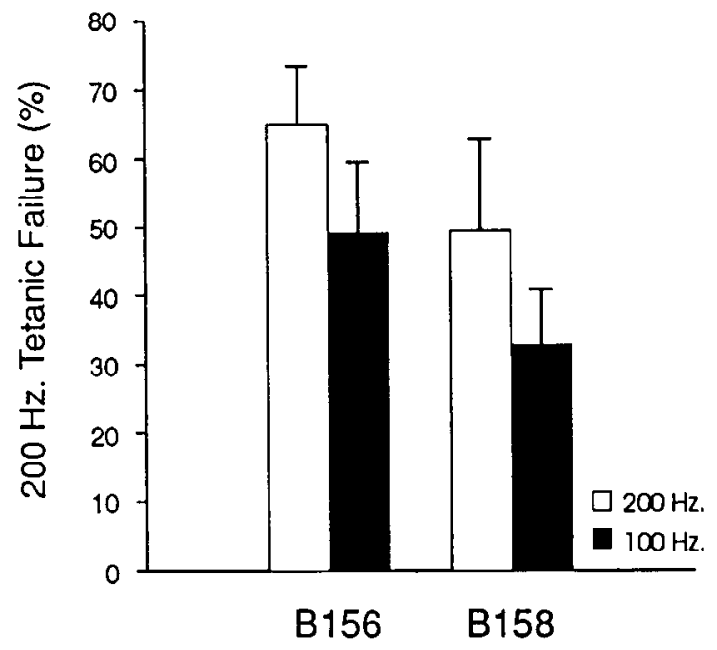

Figure 5. Failure of motor unit force in an older homozygote (B156) during high frequency motor neuron activation. $A, B$, The top records are motor unit EMG signals while the bottom row illustrates force records. $A$ shows records obtained during a tetanic stimulus of the motor neuron at $100 \mathrm{H} z$. while $B$ is for $200 \mathrm{~Hz}$. The duration of the stimulus train is indicated by the time bar shown beneath each force record. Note that the failure is increased at the higher stimulus rate. $C$, Bar chart illustrating average tetanic failure at 100 and $200 \mathrm{~Hz}$. Failure was measured as the difference between peak force and that present at the end of the intracellular train, normalized to the peak force and expressed as percent (measured points indicated by arrowheads in $A$ and $B$ ).

ron I sec later evoked a small tetanic force that failed (Fig. 6B). The twitch record and its associated EMG signal obtained after a 1 sec delay, however, were considerably enhanced (Fig. 6C). The subsequent peak tetanic force (Fig. $6 \mathrm{D}$ ) was also increased, but this force failed, the failure being associated with a rapidly decrementing unit EMG (see inset, Fig. 6D). The increased twitch force and EMG were maintained for six subsequent twitch/tetanus cycles. Despite these effects on force amplitude, the time course of the enhanced twitch contraction did not differ from the initial twitch (Fig. $6 E$ ), demonstrating that the effect did not influence muscle fiber twitch properties. The enhanced tetanic force also continued to fail with a similar time course (Fig. $6 F$ ). Since both unit EMG and force were simultaneously increased, the conditioning effect of the first tetanus was most likely to increase the number of activated muscle fibers by enhancing neurotransmission. Apparently, however, the additional neurotransmission is just as likely to fail at high activation frequency as that present before the conditioning tetanus.

Twitch potentiation was quantified by taking the difference between the amplitude of the initial twitch and that following five to nine twitch/tetanus cycles and normalizing the difference to the initial amplitude. The results (expressed as a percent of the unpotentiated amplitude) are illustrated in Figure $7 A$, which shows postnatal age plotted against mean twitch potentiation for younger homozygotes and clinically normal animals, with potentiation of failing (open triangle and square) and nonfailing units (filled triangle and square) from older homozygotes plotted separately. While mean twitch potentiation of nonfailing units is comparable to that observed in all younger animals, mean potentiation of failing units is strikingly larger. Among those units exhibiting force failure at $200 \mathrm{~Hz}$, the log of twitch potentiation was significantly correlated with the extent of failure $(r=0.74, p<0.001)$, as may be seen in Figure $7 B$. This correlation demonstrates that twitch potentiation and tetanic failure are related, the number of muscle fibers added by potentiation presumably corresponding to the number that is deactivated during the tetani. The common factor underlying these phenomena is thus likely to be abnormally labile neurotransmission.

Further analysis demonstrated that the relationship between the potentiated and unpotentiated twitch forces differed between those units that displayed tetanic failure and those that did not. This is illustrated in the three-dimensional plot of Figure 8 , which allows simultaneous consideration of the extent of tetanic failure, relative twitch potentiation, and absolute potentiated twitch amplitude all as a function of unpotentiated twitch amplitude. Among units that showed tetanic failure (filled symbols), it may be seen that a trend for decreasing tetanic failure cxists as unpotentiated twitch amplitude increases over a range of unpotentiated twitch amplitude that is comparable to nonfailing units. The extent of failure was significantly correlated with the unpotentiated twitch amplitude $(r=-0.78, p<0.001)$, indicating that the failure was proportionately greatest among motor units with initially small twitch amplitudes. Figure 8 also shows that, over a similar range of unpotentiated twitch force, failing units produced proportionately greater twitch potentiation than nonfailing units, with the largest difference present in the lower part of the unpotentiated twitch range. Since no significant differences of mean potentiation existed between the two experiments (Fig. $7 A$ ), the data were combined into separate failing and nonfailing groups. The parameters of regression equations describing the linear relationship between the logs of potentiated and unpotentiated twitch amplitudes were compared with paramcters from younger homozygous and clinically normal experiments. The results, shown in Table 2 , demonstrate that the parameters of nonfailing units are indistinguishable from the mean parameters of younger homozygous and clinically normal experiments, while those of failing units are clearly different. These results show that the twitches of smaller-force, failing units potentiate substantially more relative to both small-force, 
H

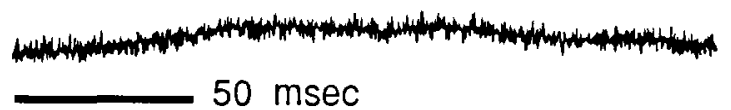

$\mathrm{B}$

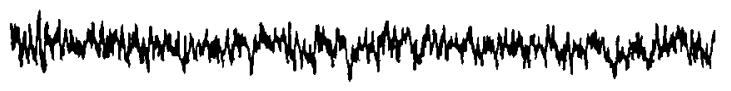

$4 \mathrm{gm}$.

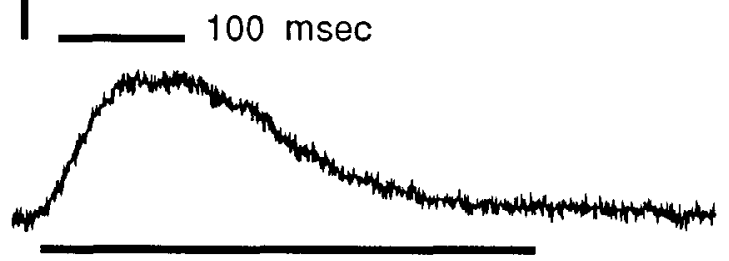

$E$

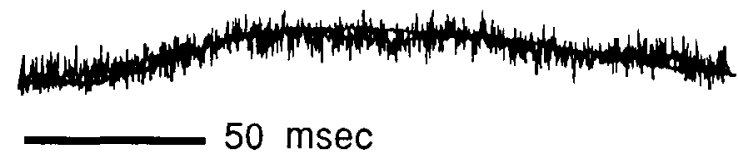

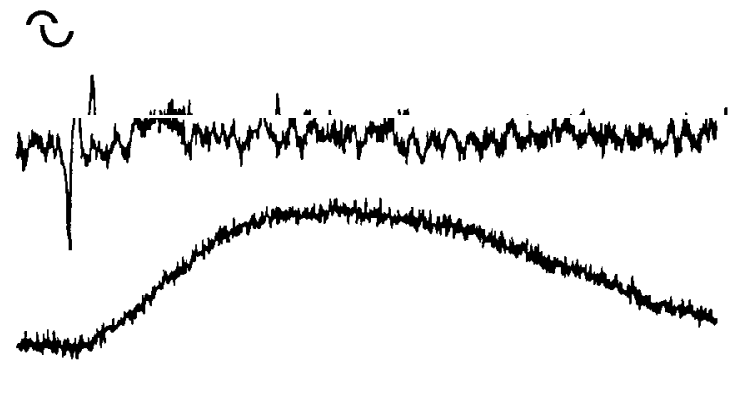

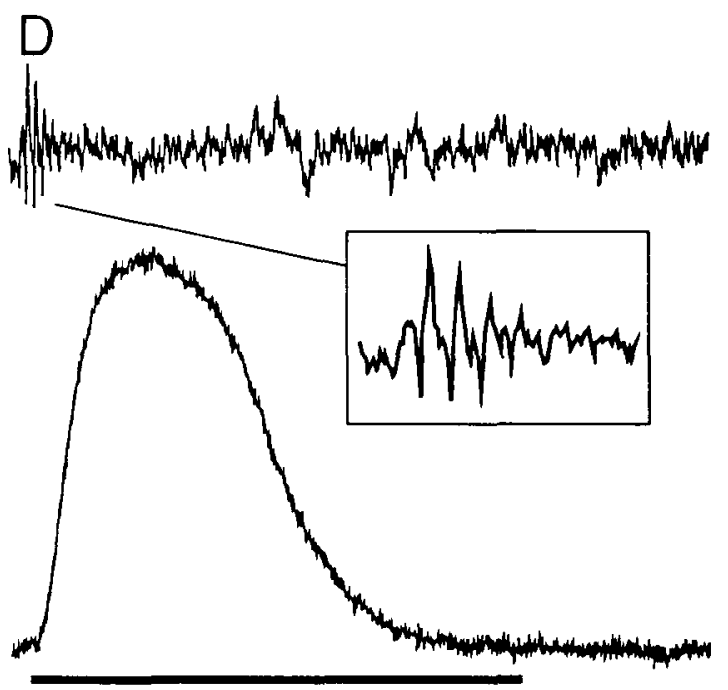

B156 M29

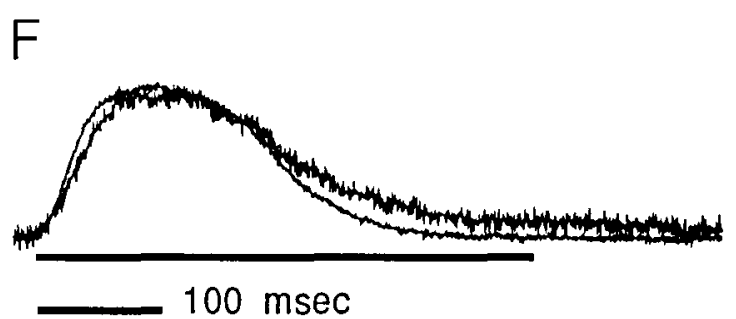

Figure 6. Potentiation of motor unit twitch force following a single tetanus. The upper records in $A-I)$ are EMG activity; lower records show motor unit force output. The top row shows twitch records and the bottom row shows tetanic force records. The duration of the motor neuron tetanic stimulus is indicated by the horizontal bars in $B$ and $D$. The records were obtained in series (separated by 1 sec) while alternately delivering single and tetanic $(150 \mathrm{~Hz})$ stimuli to the impaled motor ncuron. The first twitch $(A)$ and tetanus $(B)$ showed small forces. Note that the succeeding twitch $(C)$ and tetanus $(D)$ were markedly potentiated by the first tetanus, although failure to maintain force persisted during the second tetanus $(D)$ and was associated with a decrementing unit EMG response (inset, $D$ ). E. Twitch records of $A$ and $C$ superimposed and normalized to identical maximum amplitude. $F$, Tetanic force records of $B$ and $D$ superimposed and normalized to identical maximum amplitude. Time calibration in $A$ is for $A, C$, and $E$. Time calibration in $B$ is for $B, D$, and $F$. Force calibration in $B$ is for $A-D$.

nonfailing units and to larger-force, failing units. The results also show that those units with the largest twitch potentiation also exhibit the greatest extent of tetanic failure.

Tetanic failure and twitch speed. Another property of failing units in older homozygotes was that almost all had relatively long twitch tines to peak. This may be seen in the three-dimensional plot of Figure 9. With the exception of two units from one experiment, all failing units had potentiated twitch times to peak greater than $55 \mathrm{msec}$. One possibility is that the involvement of units at this stage of the disorder tends to make affected units contract more slowly. It may be seen, however, that no failing units possessed times to peak longer than observed for nonfailing units. The mean potentiated twitch times to peak of all units (failing and nonfailing) from each older homozygote were quite comparable with those from all younger HCSMA animals (Fig. 10A). Moreover, the proportions of units with twitch times to peak greater and less than $55 \mathrm{msec}$ in both older homozygotes were nearly identical to the mean proportions from younger homozygotes and clinically normal animals (Fig. 10B). These results indicate that tetanic failure and the associated disproportionate twitch potentiation tend to occur selectively among slowly contracting motor units. These data also show that HCSMA is not associated with alterations of muscle fiber contraction speeds. 

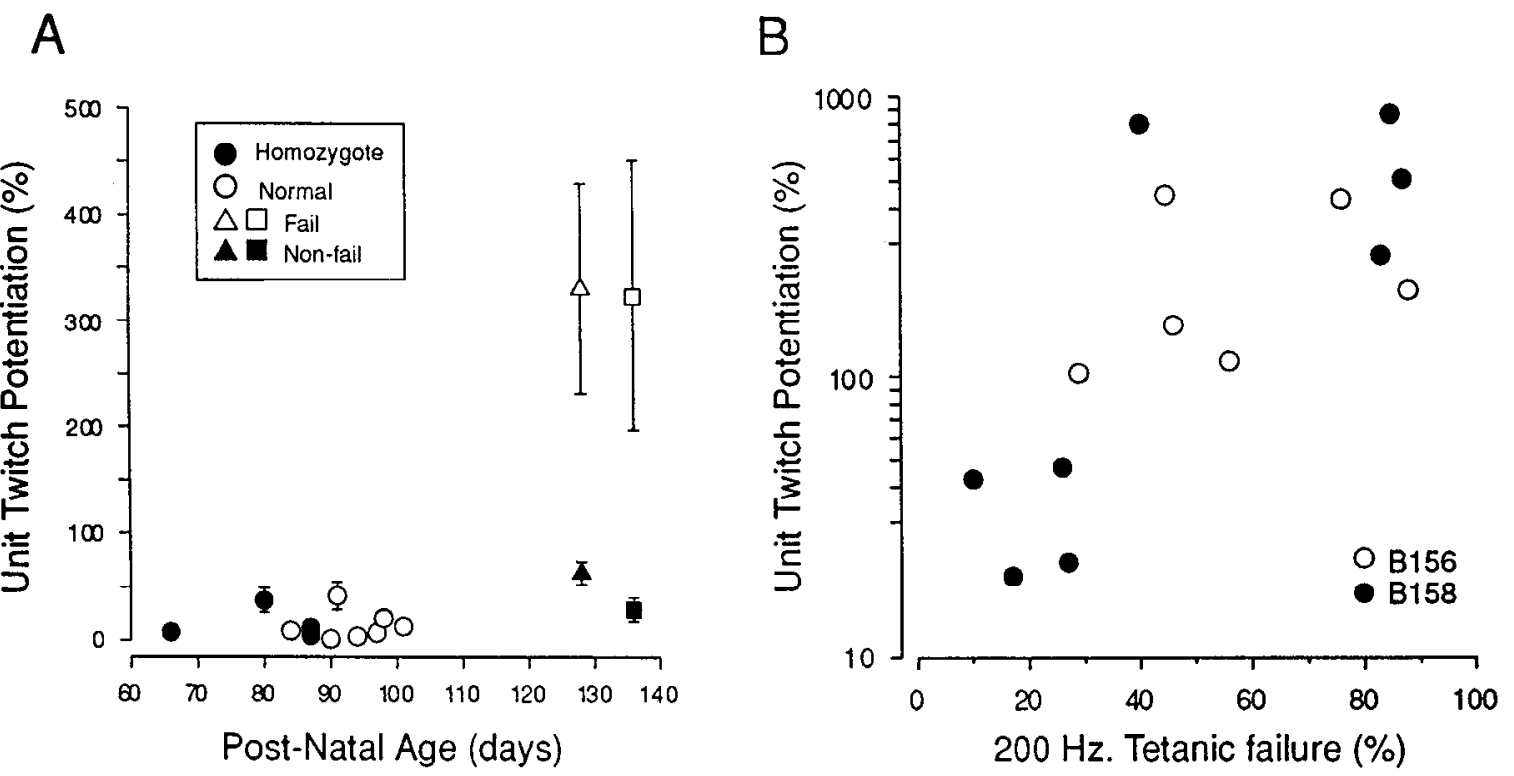

Figure 7. Plot of average unit twitch amplitude potentiation in response to preceding tetani. Solid and open circles represent homozygotes and clinically normal animals, respectivcly. For cach of the two oldest homozygotes, two values are shown: the upper values (open triangle, square; Fail) represent the average for units that showed failure to maintain tetanic force at $200 \mathrm{~Hz}$, while the lower values (filled triangle, square; Nonfail ) represent the average from units that did not show high frequency tetanic failure. $B$, Plot of twitch potentiation versus tetanic failure at 200 $\mathrm{Hz}$ for all units that displayed tetanic failure $(r=0.74, p<0.001)$. Data from older homozygotes only $(B 156, B 158)$. Vertical error bars indicate \pm 1 SEM.

\section{Discussion}

The data reported here are the first descriptions of the functional properties of fully isolated motor units in any motor neuron disease and demonstrate that HCSMA is a dynamic process that features several modes of motor unit dysfunction. In younger HCSMA homozygotes, some motor neurons fail to activate muscle fibers. Despite this, these motor neurons can be antidromically activated by nerve stimulation near the muscle and are capable of propagating action potentials to the same region. The results indicate that the site of this "disconnection" is located distal to our peripheral stimulation sites, presumably in the terminal arbor. In older homozygotes, such disconnected motor neurons were not observed. Many motor units, however, exhibited anomalous behavior such as an inability to maintain force output during tetanic activation and abnormally large potentiation of twitch contractions following tetani. These results indicate that the manner in which homozygote motor units exhibit dysfunction, and thus contribute to evolving weakness, differs as a function of postnatal age and, presumably, disease progress. Below we consider some possible mechanisms that may underlie

\section{Table 2. Regression parameters for potentiated versus} unpotentiated twitch amplitude

\begin{tabular}{llll} 
Slope $^{\prime \prime}$ & Intercept & Regression & Coefficient \\
\hline Homozygotes $^{h}$ & $0.98 \pm 0.01$ & $0.07 \pm 0.03$ & $0.95 \pm 0.02$ \\
Faik $^{\text {Nonfail }}$ & 0.48 & 0.73 & 0.94 \\
Normals $^{\prime \prime}$ & 1.05 & 0.13 & 0.97 \\
& $1.02 \pm 0.02$ & $0.03 \pm 0.03$ & $0.96 \pm 0.03$
\end{tabular}

"Fquation used: log(potentiated twitch amplitude) $=$ constant + slope $[\log ($ unpotentiated twitch amplitude)].

"Values shown for homozygotes and normals are the means of five (mean $N$ $\approx 12$ ) and seven (mean $N \approx 7$ ) individual experiments, respectively.

' $N=15$ for failing units, and $N=22$ for nonfailing units. these differences as well as factors that may contribute to the variable expression of HCSMA at the level of the motor unit.

\section{Younger homozygotes}

The main deficit observed in younger HCSMA homozygotes is an inability of some motor neurons to activate muscle. The findings are compatible with a complete absence of more distal components of the axon, conduction failure in the terminal arbor, and/or failure of transmitter release. On the basis of our data, we cannot exclude the possibility that these neurons lack a terminal arbor because they are in a growth or regenerative state and may eventually develop functional neuromuscular contact. However, several considerations argue that these neurons have in fact lost functional contact with muscle. In muscles of the tail, which are the first to become involved in HCSMA, degenerating nerve fibers and corresponding denervation atrophy of muscle fibers have been observed in young homozygotes (Alderson and Cork, 1992). While it is important to remember that proximal muscles are more extensively involved at any particular stage of HCSMA, it is probable that such degeneration eventually extends to more distally located muscles such as MG. Contributing to the notion that distal axonal degeneration is involved is the fact that some of the functional deficits we observed, as well as the apparent selectivity of distal motor nerve involvement, parallel events that occur during distal degeneration following nerve section (Lunn et al., 1990). Following nerve section in normal rats, degeneration and malfunction of motor terminals become evident before degeneration of myelinated axons (Miledi and Slater, 1971). Within hours after nerve section, evoked neurotransmission fails abruptly and this failure is associated with the onset of morphological changes in the motor terminals. Subsequently, action potential propagation fails and this is associated with morphological evidence of axonal degeneration. Our results indicate that the entire terminal arbor and/or intramuscular portion of some motor axons is functionally 


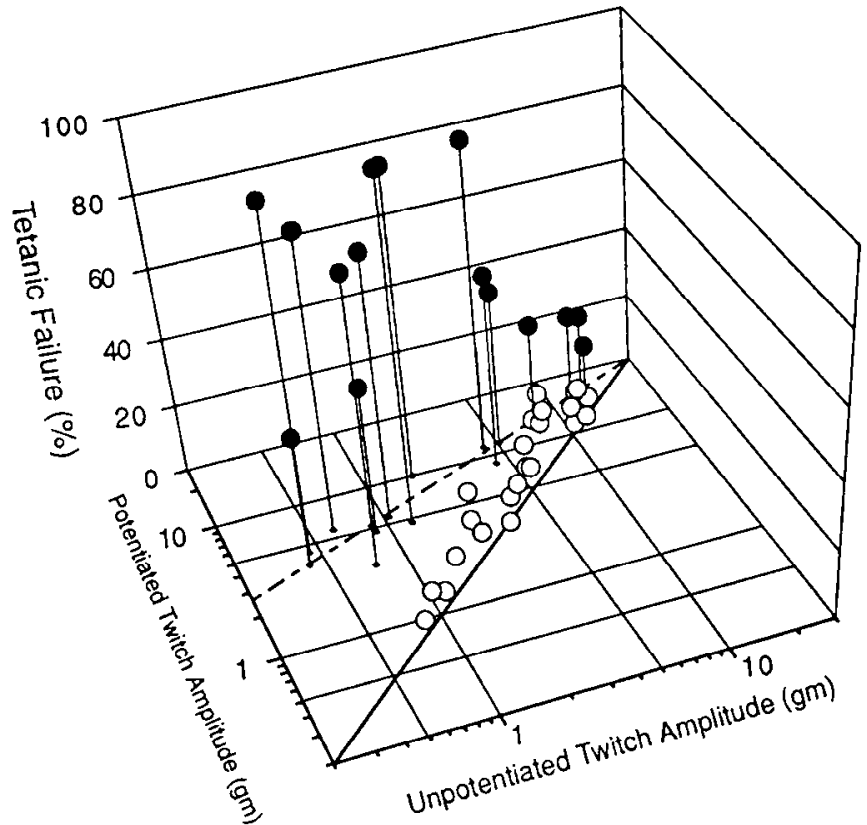

Figure 8. Plot of tetanic failure (z-axis) versus the logs of potentiated and unpotentiated twitch amplitude $(x, y$-axis) for older homozygotes (B156, B 158). Filled symbols indicate failing units; open symbols in dicate nonfailing units. The solid line drawn through the diagonal in the xy plane represents unity slope or zero twitch potentiation. Dashed line represents the least squares regression line for potentiated versus unpotentiated twitch amplitude for failing units only. Among units that display the most failure and possess smaller unpotentiated amplitudes, note disproportionately large potentiation relative to nonfailing units of similar unpotentiated amplitude.

silent or absent or that neurotransmission has otherwise totally failed, since functional connectivity of some motor neurons with muscle is completely absent. The involved motor neurons, however, remain capable of propagating action potentials in both directions along the axon at least to the level of nerve entry into muscle. The abrupt aspect of neurotransmission failure described for distal degenerating motor axons may also help explain the failure of one HCSMA motor unit during testing (Fig. 3). Overall, it seems most likely that "disconnected" motor neurons have lost functional contact with muscle and that this represents an early stage of motor unit dysfunction in HCSMA.

Several known features of HCSMA could conceivably contribute to a situation in which some of the functional aspects of nerve transection might be mimicked. Included are findings of a decreased rate of slow axonal transport (Griffin et al., 1982) and selectively decreased expression of NF-L mRNA (Muma and Cork, 1993). The latter findings may be of particular importance since recent results in transgenic mice that overexpress NF-L demonstrate that alterations in neurofilament economy can lead to some of the pathological signs of motor neuron disease (Xu et al., 1993). In HCSMA, we do not know whether these defects eventually cause motor neuron cell death or are only manifestations of a more generalized degenerative process.

\section{Older homozygotes}

In contrast to younger homozygotes, all MG motor neurons sampled in the older homozygotes were capable of activating muscle fibers and "disconnected" motor neurons were not observed. There are two possible explanations for the absence of "disconnected" motor neurons: either previously disconnected motor neurons have formed connections with muscle or they cannot be

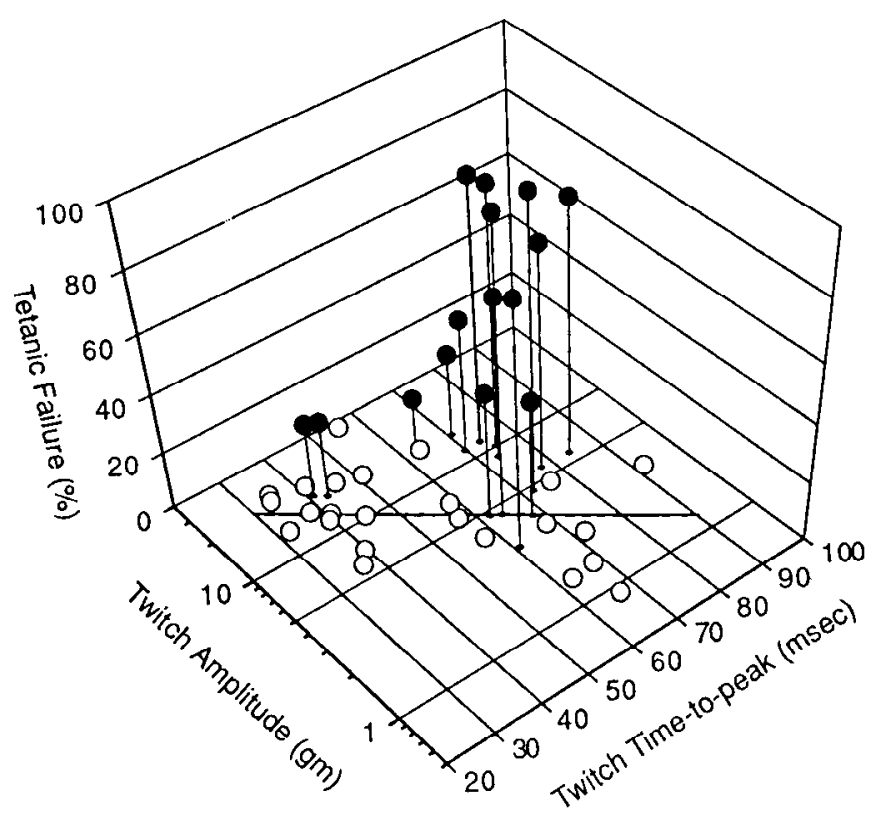

Figure 9. Plot of tetanic failure ( $x$-axis) versus the log of potentiated twitch amplitude (y-axis) and twitch time to peak (x-axis) for older homozygotes (B156, B 158). Filled symbols indicate units exhibiting tetanic failure; open symbols indicate units that did not fail. The solid line in the $x y$ plane represents the least squares regression line for the $\log$ of potentiated twitch amplitude versus twitch time to peak for nonfailing units only $(r=-0.67, p<0.001)$.

identified antidromically from the periphery because their axons have either "died back" or have otherwise become electrically inexcitable. As mentioned above, we cannot exclude the possibility that "disconnected" neurons eventually form or reform connections with muscle. Distal axon and motor terminal degeneration are known to occur in HCSMA, however, and the end result is complete denervation, at least among tail muscles (Alderson and Cork, 1992). It thus seems more likely that "disconnected" motor neurons are not present in older homozygotes because their axons have either "died back," or have become electrically inexcitable. Limited available evidence suggests that some human motor neuron diseases may be similar in featuring dying back of motor axons (Bradley, 1987).

The principal motor unit dysfunction in older homozygotes was an inability to maintain tetanic forces. This effect (1) was proportionately greatest among units producing the least unpotentiated twitch forces, (2) occurred among the slowest contracting motor units, and (3) increased at higher activation frequencies and was associated with decrementing unit EMG signals similar to that described in human motor neuron disease (Denys and Norris, 1979). Many failing units also showed disproportionately large potentiation of twitch contractions relative to nonfailing units with comparable unpotentiated twitch amplitudes, and the extent of the potentiation was correlated with the extent of tetanic failure. The fact that both twitch potentiation and tetanic failure involve marked changes of unit EMG activity suggests that alterations or defects of neurotransmission are involved. This is also indicated by the apparent frequency sensitivity of tetanic failure. The likely mechanism underlying potentiation is thus an increased number of active muscle fibers because of enhanced neurotransmission, while that of tetanic failure is a decreased number of active fibers because of failing transmission 

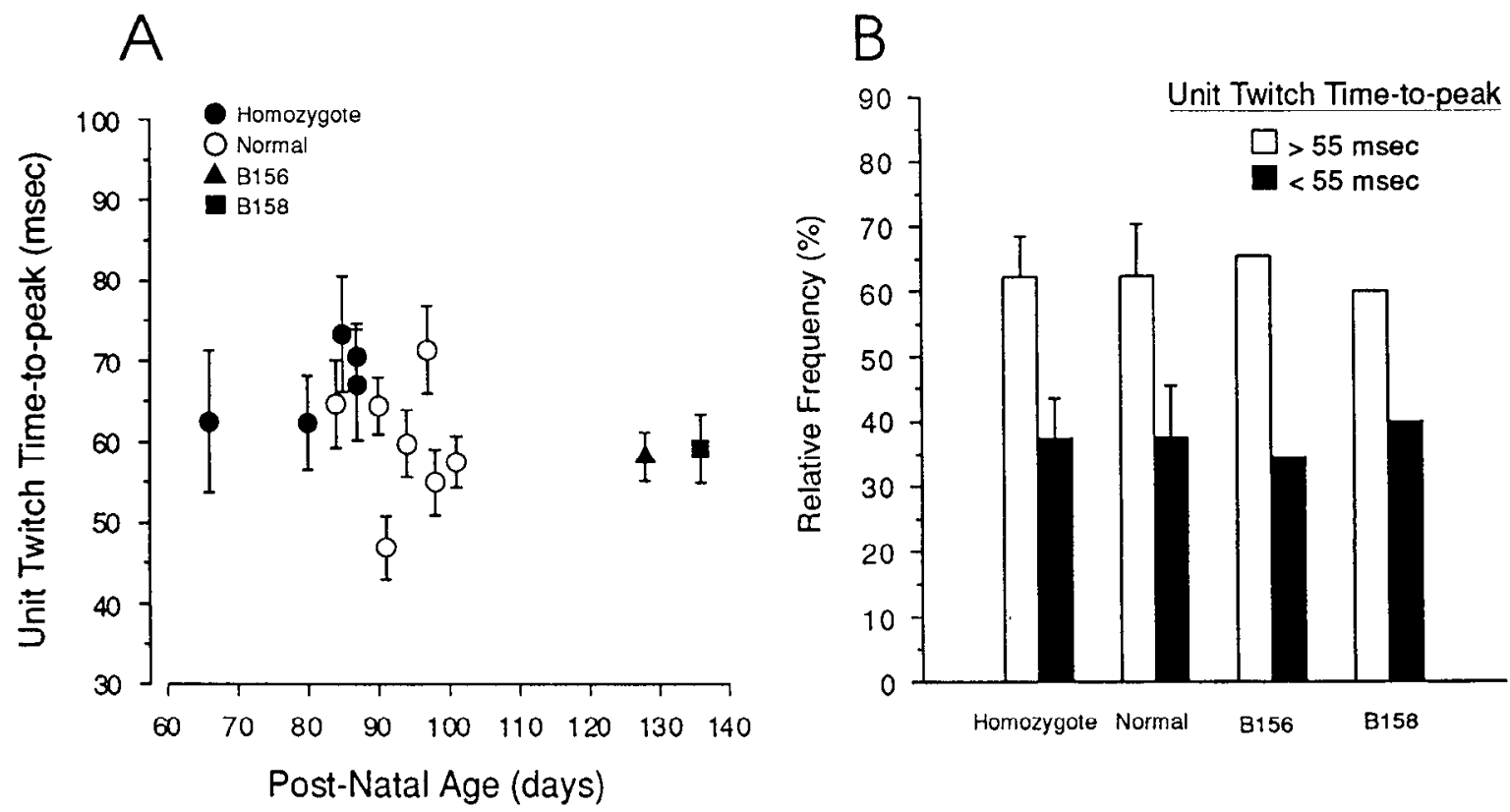

Figure 10. A. Plot of mean potentiated twitch time to peak for younger homozygotes, clinically normal animals, and older homozygotes, with symbols as indicated by inset. Multiple regression shows that neither age nor phenotype contribute significantly to the variation of mean time to peak $(p>0.80) B$, Bar chart showing proportions of motor units with twitch times to peak greater than and less than 55 msec, with bar fills as indicated by inset. Data for younger homozygotes (homozygote), clinically normal animals (normal), and older homozygotes (B156, B158). Error bars represent +1 SFM

Similar motor unit tetanic failure has been observed following partial denervation of the cat soleus muscle (Luff and Torkko, 1990). In this situation, individual motor neurons increase the number of muscle fibers innervated via nodal and/or terminal sprouting (Brown et al., 1981; Brown and Lunn, 1989), and available evidence indicates that the synapses formed by these sprouts are less reliable (Slack and Hopkins, 1982; Rochel and Robbins, 1988). Observations of unmyelinated terminal sprouts in proximal tail muscles of young homozygotes (Alderson and Cork, 1992) and in MG muscle (M. J. Pinter, R. F. Waldeck, N. Wallace, and L. C. Cork, unpublished observations) of older HCSMA homozygotes provide support for the possibility that newly formed synaptic connections exist in HCSMA. Motor terminal sprouting has been observed in human motor neuron disease as well (Alderson et al., 1989). In HCSMA, sprouting could be the result of previous muscle fiber inactivity due to the occurrence of "disconnected" motor neurons or ongoing denervation of muscle fibers. Tetanic failure may thus be related to the functional properties of newly formed axon terminals or synapses (Edds, 1953) or conduction failure in unmyelinated axonal sprouts, as observed in human motor neuron diseases (Stalberg and Thiele, 1972). A complement of newly formed but initially inactive synapses might explain why some failing units exhibit such disproportionately large twitch potentiation. It is conceivable that the relative extent of twitch potentiation among failing units provides an indirect measure of the extent of sprouting that may have occurred. If so, then our results indicate that motor neurons that have sprouted the most innervate units that fail to maintain tetanic force to the greatest extent. This possible association of motor unit failure with sprouting is of particular interest in view of speculation that sprouting of surviving neurons in human motor neuron disease might be a factor that adversely affects subsequent functional capabilities of motor neurons (Stalberg, 1982). Similar suggestions have been made regarding the role of previous sprouting in the postpolio syndrome (Trojan et al., 1993). It would therefore be of interest to determine conclusively whether tetanic failure in HCSMA is specific for units innervated by motor neurons whose terminals have sprouted.

Our results also showed that tetanic failure and large twitch potentiation tend to occur in motor units with the longest contractions times (Fig. 9). Analysis of unit contraction speed in relation to age, HCSMA phenotype, and the distribution of twitch time to peak demonstrates that this is not the consequence of any direct effect of HCSMA on motor unit contraction speed (Fig. 10). Possible explanations for this include a selective involvement of the motor neurons innervating these types of muscle fibers at this stage, a selective response of these motor neurons to other events provoked by the disorder, or a combination of factors. It may be, for example, that the terminals of these "slow-type" motor neurons sprout more readily in the presence of nearby denervated or inactive muscle fibers. This would be consistent with the disproportionate twitch potentiation displayed by these units, with the large extent of tetanic failure providing additional evidence of disease involvement. $\Lambda$ vailable evidence from normal cat MG indicates that motor axon sprouting either does not occur selectively among particular motor unit types following partial denervation (Rafuse et al., 1992) or occurs the most among type FR motor units (Luff et al., 1988). It is not known, however, to what extent such results can be applied in the normal dog or in HCSMA, and further work will be needed to resolve this issue.

\section{HCSMA expression}

Several issues regarding the expression of HCSMA are raised by our findings. One concerns the fact that involvement of the MG nucleus is not complete at any postnatal age studied thus far. An extreme example of this is the young homozygote B 170 
(Table 1) in which no MG motor unit deficits were detected despite clear evidence for proximal musculature involvement. The fact that this animal was from a third litter suggests that some interlitter variability exists in how rapidly involvement extends to motor nuclei innervating more distally located musculature. The factors that determine such timing differences between animals as well as the proximal to distal, caudal to rostral pattern of muscular involvement within animals are unidentified but probably also play a role in determining the extent of involventent within motor nuclei. The existence of such temporal and spatial gradients of involvement imply, however, that in addition to the single defective autosomal gene (Sack et al., 1984), other factors participate in controlling the expression of HCSMA. Such factors might include modifier or epistatic genes that influence transcription, translation, or activity of the defective gene product. Similar arguments can be applied for understanding why the character of HCSMA expression (i.e., "disconnected" units $v$ s tetanic failure) may vary at the level of the motor unit as a function of postnatal age. Consideration of this particular aspect of HCSMA expression should not, however, distract attention from the similarities of these motor unit dysfunctions; both involve functional defects localized to the same region (distal axonal components) that leads to the identical symptom (weakness). Clarification of the mechanisms underlying these deficits in HCSMA will provide useful insights, particularly into the more sporadically occurring varieties of human motor neuron disease and the postpolio syndrome, in which motor unit performance similar to what we describe here has been observed and similar functional issues have been raised (Denys and Norris, 1979; Stalberg, 1982; Bradley, 1987; Maselli et al., 1993; Trojan et al., 1993).

\section{References}

Alderson K, Cork LC (1992) Terminal motor axon morphology in homozygous hereditary canine spinal muscular atrophy. Soc Neurosci Abstr 18:1083.

Alderson K. Pestronk A, Lee W-C, Drachman DB (1989) Silver cholinesterase immunochemistry: a new neuromuscular stain. Muscle Nerve 12:9-14.

Bradley WG (1987) Recent views on amyotrophic lateral sclerosis with emphasis on electrophysiological studies. Muscle Nerve 10:490-502.

Brown MC (1984) Sprouting of motor nerves in adult muscles: a recapitulation of ontogeny. Trends Neurosci 7:10-14.

Brown MC. Lunn ER (1988) Mechanism of interaction between motor neurons and muscles. In: Plasticity of the neuromuscular system, pp 78-96. Chichester: Wiley.

Burke RE (1981) Motor units: anatomy, physiology and functional organization. In: Handbook of physiology, the nervous system, motor control (Brooks VB, ed), pp 345-422. Bethesda: American Physiological Society.

Burke R. Levine DN, Tsairis P, Zajac FE (1973) Physiological types and histochemical profiles in motor units of cat gastrocnemius. J Physiol (Lond) 234:723-748.

Cork LC, Griftin JW, Munnell JF, Loren\% MD, Adams RJ, Price DL (1979) Hereditary canine spinal muscular atrophy. J Neuropathol Exp Neurol 38:209-221.

Cork LC, Griftin JW, Choy C, Padula CA, Price DL (1982) Pathology of motor neurons in accelerated hereditary canine spinal muscular atrophy. Lab Invest 46:89-99.
Cork LC, AItschuller RJ, Bruha PJ, Morris JM, Lloyd DG, Loats HL, Griffin JW, Price DL (1989a) Changes in neuronal size and neurotransmitter marker in hereditary canine spinal muscular atrophy. Lab Invest 61:69-76.

Cork LC, Struble RG, Gold BG, DiCarlo C. Fahenstock KE, Griffin JW, Price DL (1989b) Changes in the size of motor axons in Hereditary Canine Spinal Muscular Atrophy, Lab Invest 61:33.3-342.

Denys EH, Norris FH (1979) Amyotrophic lateral sclerosis. Impairment of neuromuscular transmission. Arch Neurol 36:202-205.

Edds MV (1953) Collateral nerve regeneration. Q Rev Biol 28:260276.

Griffin JW, Cork LC, Adams RJ, Price DL (1982) Axonal transport in hereditary canine spinal muscular atrophy (HCSMA). J Neuropathol Exp Neurol 41:370.

Hays WL (1988) Statistics. New York: Holt, Rinehart and Winston.

Hoffman PN, Griffin JW, Price DL (1984) Control of axonal caliber by neurofilament transport. J Cell Biol 99:705-714.

Hoffman P, Koo EH, Muma NA, Griftin JW, Price DL (1988) Role of neurofilaments in the control of axonal caliber in myelinated nerve fibers. In: Intrinsic determinants of neuronal form and function, pp 389-402. New York: Liss.

Luff AR, Torkko K (1990) Long term persistence of enlarged motor units in partially denervated hindlimb muscle of cat. $\mathbf{J}$ Neurophysiol 64:1261-1269.

Luff AR, Hatcher DD. Torkko K (1988) Enlarged motor units resulting from partial denervation of cat hindlimb muscles. J Neurophysiol 59: 1377-1394.

Lunn ER, Brown MC, Perry VH (1990) The pattern of axonal degeneration in the peripheral nervous system varies with different types of lesion. Neuroscience 35:157-165.

Maselli RA, Wollman RL, Leung C, Palombi S, Richman DP, SlalzarGrueso EF, Roos RP (1993) Neuromuscular transmission in amyotrophic lateral sclerosis. Muscle Nerve 16:1193-1203.

Muma NA, Cork LC (1993) Alterations in neurofilament mRNA in hereditary canine spinal muscular atrophy. Lab Invest 69:436-442.

Pinter MJ, Vanden Noven S, Muccio D, Wallace N (1991) Axotomylike changes in cat motor neuron electrical properties elicited by botulinum toxin depend on the complete elimination of neuromuscular transmission. J Neurosci 11:657-666.

Pinter MJ, Cork LC, Wallace N (1992) Motor unit properties in Hereditary Canine Spinal Muscular Atrophy. Soc Neurosci Abstr 18: 1083.

Rafuse VF, Gordon T, Orozco R (1992) Proportional enlargement of motor units after partial denervation of cat triceps surae muscles. $J$ Neurophysiol 68:1261-1276.

Rochel S, Robbins N (1988) Effect of partial denervation and terminal field expansion on neuromuscular transmitter release and nerve terminal structure. J Neurosci 8:332-338.

Sack GH Jr, Cork LC, Morris JM, Griffin JW, Price DL (1984) Autosomal dominant inheritance of hereditary canine spinal muscular atrophy. Ann Neurol 15:369-373.

Slack JR, Hopkins WG (1982) Neuromuscular transmission at terminals of sprouted mammalian motor neurones. Brain Res 237:121135.

Stalberg E (1982) Electrophysiological studies of reinnervation in ALS. In: Human motor neuron disease (Rowland LP, ed), pp 47-59. New York: Raven.

Stalberg E, Thiele B (1972) Transmission block in terminal nerve twigs: a single fibre electromyographic finding in man. J Neurol Neurosurg Psychiatry 35:32-59.

Trojan DA, Gendron D, Cashman NR (1993) Stimulation frequencydependent neuromuscular junction transmission defects in patients with prior polimyelitis. J Neurol Sci 118:150-157.

Xu Z, Cork LC, Griffin JW, Cleveland DW (1993) Increased expression of neurofilament subunit NF-L produces morphological alterations that resemble the pathology of human motor neuron disease. Cell 73:23-33. 